\title{
La descente testiculaire
}

\author{
E. VENDRELY \\ Laboratoire de Biologie de la Fertilité et de Cytogénétique, Place du Parvis Notre-Dame \\ 75181 Paris Cedex 04
}

\section{RESUME}

La formation des gonades est intimement liée à celle du mésonéphros ou rein intermédiaire. Les testicules apparaissent dans la région dorsale de l'embryon, en arrière de la cavité coelomique abdominale. Pour gagner leur situation définitive les testicules descendent dans la cavité pelvienne, franchissent la paroi abdominale par l'anneau inguinal et se fixent au fond de la poche scrotale. La migration testiculaire se fait ainsi en trois phases successives ayant chacune leur mécanisme propre. Les organes annexés aux testicules (épididyme et déférent) et surtout les ligaments qui les relient à la paroi (gubernaculum testis) jouent un rôle essentiel dans ces déplacements.

Mots-clés : Cryptorchidie, embryologie, epididyme, gubernaculum testis, migration testiculaire, testicule.

\section{INTRODUCTION}

Chez pratiquement tous les mammiferres, les testicules apparaissent dans la région dorsale de l'abdomen puis migrent et sont extraabdominaux dès la naissance. Toutefois le défaut de migration, dont la conséquence est la cryptorchidie, est l'anomalie la plus fréquemment observée au cours de la différenciation sexuelle de l'homme. En effet la descente du testicule est anatomiquement com- plexe et son contrôle hormonal reste encore mal connu [14] ; les données morphologiques associées aux observations cliniques et expérimentales ont permis de décrire les grandes lignes de ce processus. Cependant de nombreuses incertitudes persistent et les causes de la cryptorchidie sont loin d'être élucidées.

\section{FORMATION DES TESTICULES}

La formation des testicules est intimement liée au développement du mésonéphros. Ils apparaissent dans la région dorsale de l'embryon, en arrière de la cavité coelomique abdominale [4].

\section{Gonade indifférente}

Dans l'embryon humain de 30 jours apparaissent les premiers éléments de la gonade, identiques dans les deux sexes (stade dit indifférent). Il s'agit d'un épaississement bilatéral de l'épithélium coelomique, dans la région dorsale, entre le mésonéphros et la racine du mésentère [13]. Ces formations, les crêtes génitales, résultent de la prolifération des cellules épithéliales coelomiques qui forment des cordons qui s'enfoncent dans le mésenchyme sous jacent. Simultanément les cellules mésoblastiques de certains néphrons du mésonéphros prolifèrent également et les cellules mésonéphrotiques viennent se mêler aux cellules coelomiques. L'ensemble constitue le blastème gonadique qui est à l'origine des cellules de Sertoli [15]. 
En même temps que se produit cette prolifération, les crêtes génitales sont envahies par les cellules germinales primordiales ou gonocytes primordiaux. Ce sont de grandes cellules claires à noyau vésiculeux qui apparaissent vers le 20 e jour dans la paroi caudale du lécithocèle (sac vitellin). Elles migrent grâce à des mouvements actifs à travers le mésenchyme péri-vitellin, passent dans la racine $\mathrm{du}$ mésentère et colonisent les crêtes génitales au cours de la cinquième semaine $\mathrm{du}$ développement. Elles se mêlent aux cellules du blastème gonadique. Les gonocytes primordiaux sont les futures cellules germinales [12].

Des cellules mésenchymateuses entourent la masse du blastème et des gonocytes: elles fournissent le troisième composant de la gonade, constitué par les cellules péri tubulaires, le tissu conjonctivo-vasculaire et la glande interstitielle de Leydig.

\section{DIFFERENCIATION TESTICULAIRE}

Vers la fin de la sixième semaine la différenciation testiculaire est marquée par l'apparition de membranes basales qui fragmentent le blastème gonadique en cordons séminifères pleins [9]. Les cellules germinales incluses dans ces cordons persisteront, celles qui sont en dehors dégénèrent. L'ensemble du blastème gonadique perd ses connexions avec la surface coelomique, des fibroblastes s'interposant pour former l'albuginée.

Les cordons testiculaires les plus profonds, d'origine essentiellement mésonéphrotique, constituent le rete testis qui se canalise en même temps que les cordons séminifères. Au cours du $4 \mathrm{e}$ mois la continuité canalaire est établie entre les cordons séminifères et le canal mésonéphrotique de Wolff grâce à 10 à 12 tubules mésonéphrotiques qui deviennent les canaux (ou cônes) efférents.

\section{GUBERNACULUM TESTIS}

La région caudale du lécithocèle donne naissance à un diverticule entoblastique, I'allantoïde, et au sinus urogénital. Cette région reçoit les canaux excréteurs des mésonéphros ou canaux de Wolff. Chacun de ces canaux est contenu dans un épaississement mésenchymateux de la paroi dorsale du coelome intraembryonnaire. Ce cordon uro-génital, qui renferme également le canal de Muller, est revêtu par l'épithélium coelomique. Il forme depuis la région dorsale jusqu'à la région pelvienne une colonne mésenchymateuse au sommet de laquelle est juché le testicule. Cette colonne, qui fait partie de la paroi dorsale, s'attache également à la paroi ventrale à sa base, dans la région inguinale. Lorsque le mésonéphros régresse, ce ligament inguinal prend le nom de gubernaculum testis.

Le mésenchyme de la paroi pelvienne prolifêre de chaque côté de la ligne médiane et forme les replis labio-scrotaux, origines des grandes lèvres dans le sexe féminin et, après soudure, du scrotum chez le mâle. L'extrémité distale du gubernaculum est incluse dans le mésenchyme sous-cutané de cette région. Le gubernaculum traverse la lame musculaire de la paroi abdominale, formant ainsi un orifice arrondi rempli d'un mésenchyme peu différencié, ébauche du canal inguinal (Figure 1). Dans la partie périphérique du mésenchyme gubernaculaire apparaissent des cellules musculaires striées qui forment le crémaster. Celui-ci est totalement indépendant des muscles abdominaux par son origine et son innervation [16].

Le mésenchyme du gubernaculum testis est très indifférencié et très lâche. Sa structure est similaire à celle de la gelée de Wharton du cordon ombilical. Il est pénétré par une évagination de la paroi cœlomique qui forme un anneau allongé incomplet de clivage entre un axe relié à la paroi dorsale par un mince méso et une enveloppe cylin- 
drique externe en continuité avec la paroi scrotale. La cavité ainsi délimitée ou processus vaginal est l'ébauche de la future cavité vaginale.

La portion terminale du gubernaculum n'est pas pénétrée par le processus vaginal. Cette portion dite infra-vaginale constitue un solide point d'ancrage sur la paroi du repli scrotal.

\section{DESCENTE TESTICULAIRE}

La migration testiculaire depuis la région para-rénale jusqu'au fond du scrotum s'effectue en trois phases. Le détail de leur mécanisme reste cependant assez hypothétique. La première phase paraît passive, indépendante de tout contrôle hormonal et peu différente dans les deux sexes. Elle amène les gonades dans la région pelvienne. Les phases suivantes, propres au testicule, dépendent de modifications du gubernaculum, ou réaction gubernaculaire, liées aux conditions hormonales propres au sexe masculin [7].

\section{Phase 1 : descente intra-abdominale}

Le gubernaculum et le processus vaginal croissent beaucoup plus lentement que la paroi abdominale correspondante. De ce fait on observe une diminution relative de la distance qui sépare le testicule de l'orifice interne du canal inguinal (Figure 2). Les vaisseaux testiculaires, dont l'origine par rapport aux reins n'est pas modifiée, s'allongent considérablement. Dès la fin du troisième mois de la vie intra-utérine, le testicule, partiellement recouvert par le péritoine, vient se placer dans l'angle dièdre formé par les parois antérieure et postérieure du bassin, à l'entrée de l'orifice interne du canal inguinal. Il demeure dans cette situation jusqu'au 7e mois [11].

\section{Phase 2 : traversée du canal inguinal}

Le transit du testicule le long du canal inguinal se produit pendant le $7 \mathrm{e}$ mois de la gestation. Plusieurs facteurs permettent cette progression, en particulier la dilatation de l'anneau inguinal par l'augmentation de volume du gubernaculum et l'accroissement de la pression intra-abdominale qui refoule le testicule vers l'extérieur [2].

Le mésenchyme gubernaculaire périphérique, lié à la paroi scrotale, est envahi par des cellules coelomiques qui prolifèrent [16]. La concentration en acide hyaluronique de la matrice extra-cellulaire du mésenchyme de cette région augmente considérablement, provoquant un appel d'eau [5]. Le mésenchyme forme alors une masse volumineuse qui distend le scrotum et élargit l'anneau inguinal [1]. Rattaché à la paroi scrotale, il ne subit pas la même évolution que le mésenchyme pariétal. Le processus vaginal l'isole de l'axe du gubernaculum lié au testicule par l'intermédiaire de l'épidididyme, Le mésenchyme axial ne subit pas les mêmes modifications que la portion périphérique : il ne s'hydrate pas et ne présente pas de dilatation.

L'anneau inguinal étant élargi par le gonflement de la portion périphérique du gubernaculum, le testicule peut le franchir facilement (Figure 3), poussé vers la cavité scrotale par l'augmentation de la pression abdominale, conséquence en particulier du développement de la musculature. Le testicule glisse le long de la paroi dorsale, au sein d'un mésenchyme très lâche. Il est recouvert sur ses faces ventrale et latérales par l'épithélium coelomique du processus vaginal.

\section{Phase 3 : fixation en position scrotale}

Lorsque le testicule a achevé la traversée du canal inguinal, on observe une régression progressive du volume du gubernaculum (Figure 4). La plus grande partie du matériel extra-cellulaire disparaît et le mésenchyme devient fibreux. La portion axiale se raccourcit considérablement. Cette évolution est tardive, survenant chez l'homme au cours du neuvième mois de la gestation. 


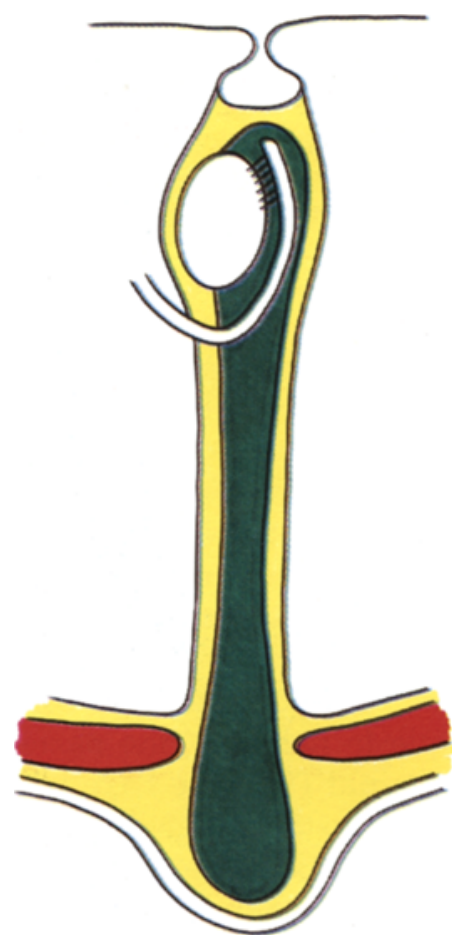

Figure 1 : Le testicule est en situation abdominale dans un canal formé par un repli du péritoine. Il est indirectement relié à la fossette inguinale par le mésenchyme du cordon uro-génital (en vert). Ce mésenchyme est l'ébauche du gubernaculum testis. Il traverse le futur canal inguinal qui forme un orifice dans les muscles du plancher pelvien (en rouge).

Le rôle de l'épididyme dans le mécanisme de la descente testiculaire est discuté. Pour certains auteurs il est très important, comme semble le montrer la fréquence de l'association de cryptorchidie et malformations épididymaires [3]. Avant le 8e mois de la gestation le canal mésonéphrotique n'est pas encore bien différencié anatomiquement en épididyme et déférent. La portion juxta-testiculaire du canal s'allonge considérablement mais en même temps se spiralise entraînant le raccourcissement de l'épididyme. Or le mésenchyme qui entoure le canal mésonéphrotique et devient dans sa portion céphalique le tissu conjonctif de l'épididyme appartient au gubernaculum.

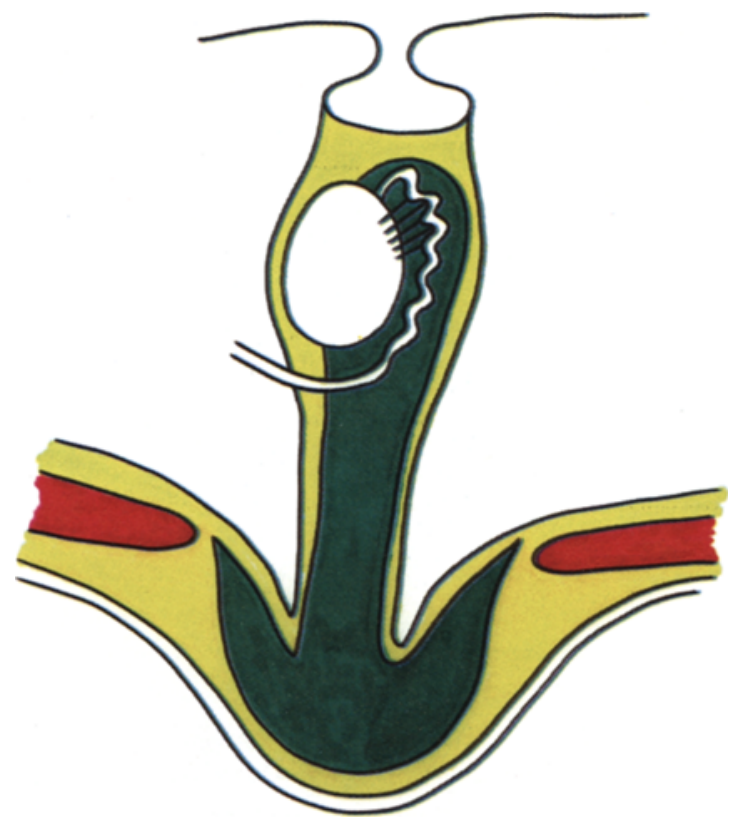

Figure 2 : Phase 1 de la descente testiculaire: le testicule se rapproche du canal inguinal par croissance différentielle du gubernaculum et de la cavité abdominale. La portion scrotale périphérique du gubernaculum s'élargit. Entre la partie axiale et la région externe du gubernaculum se forme une dépression, le processus vaginal.

Sa condensation autour des spires du canal épididymaire semble participer à son raccourcissement. Le mésenchyme forme ainsi un ligament court et solide entre l'épididyme et le testicule et relie cet ensemble au fond du scrotum [8]. L'épididyme précéderait le testicule dans le scrotum [6]. Plusieurs études mettent en évidence une grande fréquence des malformations épididymaires en liaison avec la cryptorchidie [10].

Lorsque la descente testiculaire est achevée, survient la fermeture du processus vaginal. Chez la majorité des individus, la communication entre cavité de la vaginale 


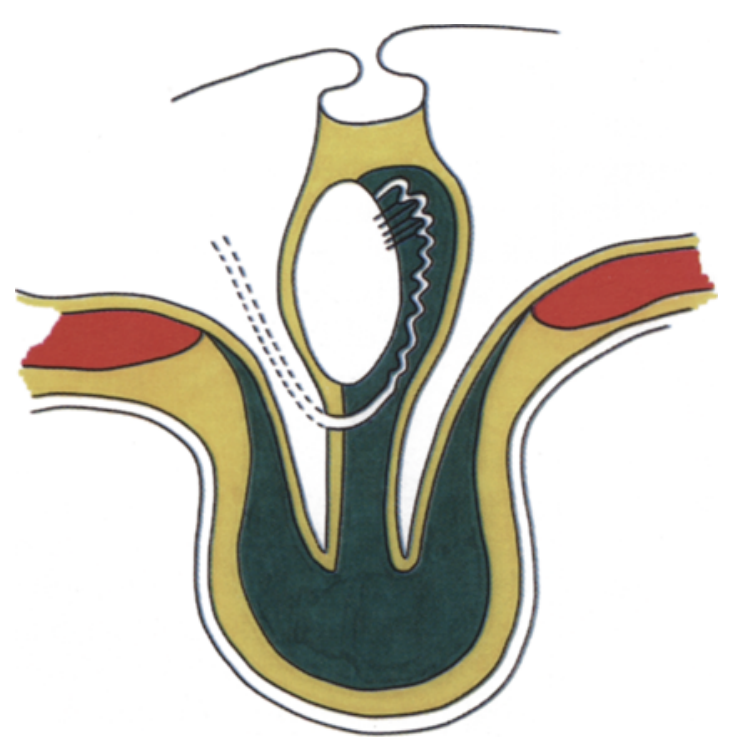

Figure 3 : Phase 2 de la descente testiculaire: la portion périphérique du gubernaculum, dilatée, élargit l'anneau inguinal. La portion axiale se raccourcit et le testicule franchit l'anneau inguinal.

et cavité péritonéale est interrompue. Toutefois cette fermeture est fréquemment imparfaite, expliquant la grande fréquence des hernies inguinales.

\section{REFERENCES}

1. BACKHOUSE K.M. : Development and descent of the testis. Eur. J. Pediatr.,1982, 139 : 249-252.

2. GIER H.T., MARION GB. : Development of mammalian testis and genital ducts. Biol. Reprod., 1969,1 : 1-23.

3. HADZISELIMOVIC F., KRUSLIN E. : The role of the epididymis in descensus testis and the topographical relationship between the testis and epididymis from the sixth month of pregnancy until immediately after birth. Anat. Embryol.,1979,155: 191-196.

4. HAMILTON W.J., MOSSMAN H.W. : Human embryology. 4th ed. Williams and Wilkins, London, 1972.

5. HEYNS C.F., HUMAN HJ., WERELY C.J., DE KLERK D.P.: The glycosaminoglycans of the gubernaculum during testicular descent in the fetus. J. Urol., 1990,143, 612-617.

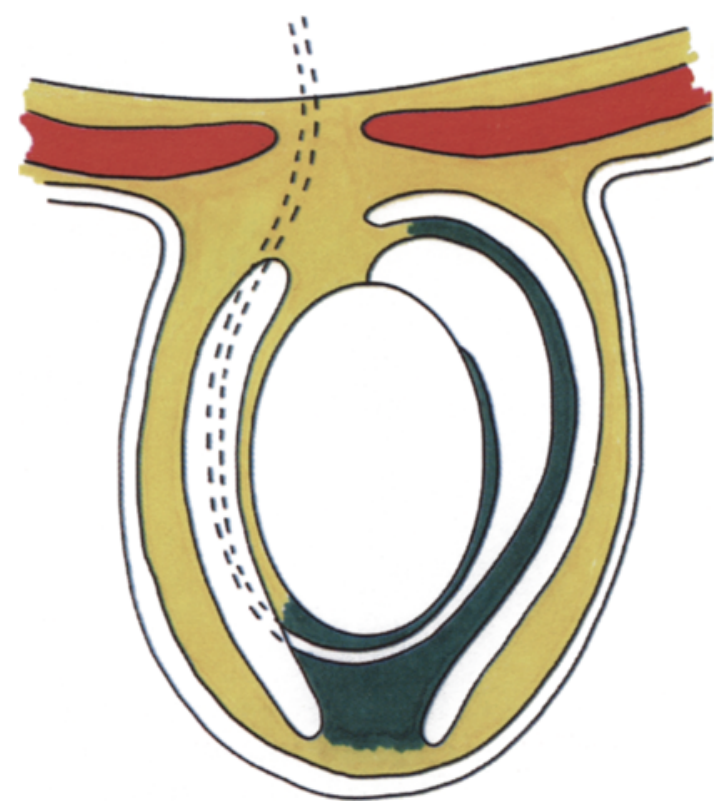

Figure 4 : Phase 3 de la descente testiculaire: le gubernaculum régresse et forme un court ligament qui fixe le testicule et l'angle épididymo-déférentiel au fond du scrotum.

6. HOLSTEIN A.F. : Morphologische Studien am Nebenhodens des Menschen. Zwangl. Abh. Geb. norm. path. Anat.1969, $20: 1-91$.

7. HUTSON J.M. : A biphasic model for the hormonal control of testicular descent. Lancet, 1985 : Il, 419-422.

8. LUDWIG K.S. : The development of the caudal ligaments of the mesonephros and of the gonads : a contribution to the development of the human gubernaculum (Hunter). Anat. Embryol.,1993, $188: 571-577$.

9. MAGRE S., JOST A. : The initial phases of testicular organogenesis in the rat. An electron microscopy study. Arch. Anat. micr. Morph. exp., 1980 : 69.297-317.

10. MOLLAEIAN M., MEHRABI V., ELAHI B. : Significance of epididymal and ductal anomalies associated with undescended testis : study in 652 cases. Urology, 1994, $43: 857-860$.

11. MOORE K.L. : The developing human. Clinically oriented embryology. W. B. Saunders, Philadelphia,1988.

12. RAJFER J., WALSH P.C. : Hormonal regulation of testicular descent: experimental and clinical observations. J. Urol.7 1977 : 118, 985-990. 
13. SATOH M. : Histogenesis and organogenesis of the gonad in human embryos. J. Anat.,1991,177 : 85-107.

14. SHAFIK A. : Epididymal ligaments : anatomy and function. Internat. J. Fertil.,1987 : 32, 324-330.

15. WARTENBERG H. : Differenciation and development of the testes. In: Burger H., de Kretser D. eds. The testis. Raven Press, New York,1989: 67-118.

16. WENSING C.J.G. : The embryology of testicular descent. ldorm. Res.,1988, 30, 144-152.

\section{ABSTRACT \\ Testicular descent \\ E. VeNDRELY}

Gonadal development is intimately linked to that of the mesonephros. The primordial testes differentiate in the dorsal region of the embryonic abdomen, behind the coelomic cavity. To reach their final location within the scrotum, the testes descend into the pelvis, pass through the abdominal wall by the inguinal canal, and attach to the base of the scrotal pouch. Testicular migration therefore comprises three stages, each requiring its own mecanism(s). The genital ducts (epididymis, vas deferens) and ligament which attaches the testis to the scrotal wall (gubernaculum testis) certainly play an essential role in these migratory events. The first phase is quite passive, associated with the growth of the abdominal cavity. In the second phase, the gubernaculum enlarges and shortens, pulling the testis through the inguinal canal. Finally, the gubernaculum, as a short, fibrous ligament, attaches the testis to the scrotal wall.

Key words : Cryptochidism, embryology, epididymis, gubernaculm testis, testis, testicular descent. 